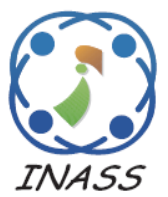

\title{
A Robust Image Enhancement Techniques for Underwater Fish Classification in Marine Environment
}

\author{
Ricardus Anggi Pramunendar ${ }^{1,2} \quad$ Sunu Wibirama ${ }^{1 *} \quad$ Paulus Insap Santosa $^{1}$ \\ Pulung Nurtantio Andono ${ }^{2}$ Moch Arief Soeleman ${ }^{2}$ \\ ${ }^{1}$ Department of Electrical Engineering and Information Technology, Faculty of Engineering, \\ Universitas Gadjah Mada, Yogyakarta, 55281, Indonesia \\ ${ }^{2}$ Department of Informatics Engineering, Faculty of Computer Science, \\ Universitas Dian Nuswantoro, Semarang 50131, Indonesia \\ * Corresponding author's Email: sunu@ugm.ac.id
}

\begin{abstract}
From literature reviews, the marine environment influences the quality of underwater images and makes the identification of fish species more complex and challenging. The images of the marine environment have low image quality that causes the generated features to be reduced; therefore, this decreases the performance of the classification method. To the best knowledge of the authors, we found out that many researchers have focussed only on determining identification methods without considering the quality of the original data. Therefore, the impact of image enhancement toward the accuracy is yet to be known because this has not been studied comprehensively. To deal with this research gap we propose a new workflow of fish species identification. The workflow for our proposed approach is by using the gray-level co-occurrence matrix (GLCM) feature extraction fed into the back-propagation neural network (BPNN) with contrast-adaptive color correction technique (NCACC) as image enhancements. The experiments demonstrated an improvement in accuracy and kappa measurements for fish species identification from $4.68 \%$ to $93.73 \%$ and improve from 0.05 to 0.92 respectively. Therefore, our proposed method has the potential to support automatic fish identification systems based on computer vision technology.
\end{abstract}

Keywords: Image enhancement, NCACC, GLCM, BPNN.

\section{Introduction}

Protecting the populations of endangered aquatic biota is very challenging for many countries. According to World Bank data, the total number of species of endangered fish in 2018 had reached 8233 species [1]. The growing number of extinct fish species has been due to increasing human needs and environmental stresses, such as a large number of migratory or invasive fish species that disturb native fish populations. Some efforts to overcome the damage caused by invasive fish species have been done by reducing population of the invasive fish through various traditional fishing techniques. However, the fishing techniques catch not only the invasive fish species but also the native species.
Therefore, a manual process to sort out the catch is still performed by distinguishing the native species from the invasive species, which involves a lot of energy and time.

On the other hand, not all types of fish biota can be manually identified by fishermen through their visual senses [2]. Manual identification by the human is prone to unexpected bias that affects the classification results. Therefore, automatic monitoring and classification of native and invasive species based on artificial intelligence technology can have a significant impact on biologists, governments, and fishers.

Current techniques of monitoring and automatically classifying objects in marine ecosystems have applied computer vision 


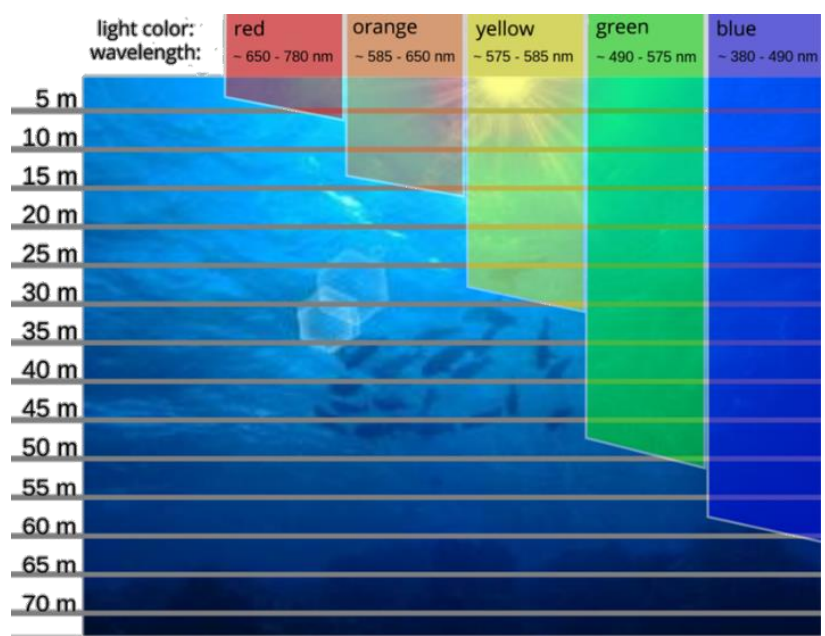

Figure. 1 Color appearance in underwater environment

technology [3]. The computer vision technology has been implemented in various sectors, such as in aquaculture where that application uses the backpropagation neural network (BPNN) method to classify marine fish species [4]. They use this technology to analyze and to recognize certain underwater objects from images and videos data obtained using sensors. However, this technology depends on the environment and the observed objects [5]. Accurate observations can be achieved if the source is in noise-free environment [6]. However, the condition of the underwater ecosystem is different from that of in the air where raw data can be adjusted to minimize noises. The characteristics of the data origin influence the data, and the data originated from underwater are controlled by the aspects of water. In this case, the characteristics of the water differ significantly compared with the characteristics of the air [7]. Therefore, appropriate setting should be made in the classification process to achieve high accuracy. The medium density level is one of the distinguishing features of water and air [8]. The water density is higher than the air; therefore, the light in the water is refracted away from the standard line. The molecules contained in the water absorb a certain amount of light. At the same time, when the light moves from the air to the surface of the water, parts of the light are reflected, and the other parts penetrate through the surface. As shown in Fig. 1, the amount of light in the water gradually decreases as the depth increases [9].

As the water depth increases, the colors' quality, brightness, contrast, and visibility decrease. The images that are taken in the deeper water also appear foggy. Some researchers solve this problem by using the dark channel prior (DCP) technique proposed by Carlevaris-Bianco et al. [10], which is further continued by Wen et al. [11] and have been updated by Kaur et al. [12], Galdran et al. [13], Borker et al.
[14], Gu et al. [3] and Pramunendar et al. [7]. However, previous works on the improvement of DCP were not able to explain the effect of improvement on the performance of computerassisted vision-based classification for objects that are affected by underwater environmental issues. On the other hand, the accuracy of marine observations is also influenced by the quality of image resolution obtained by the sensor [15] and the image quality [16]. In addition, low-quality data will have a severe impact when they are applied to traditional classification methods [16] or unlimited number of data [6].

To deal with this research gap, we propose a new workflow of fish species identification. The workflow consists of the gray-level co-occurrence matrix (GLCM) feature extraction fed into the backpropagation neural network (BPNN) with contrastadaptive color correction technique (NCACC) as image enhancements technique. The reliability of the NCACC technique on improving image quality is determined by Pramunendar, et al. [7]. However, the reliability NCACC combined with methods of feature extraction and classification methods is unknown. Therefore, we compared to the existing technique for image enhancement, such as dark channel prior (DCP), automatic level color correction (ALCC), limited adaptive limited histogram equalization (CLAHE), automatic white balance (AWB), gamma correction (GC) and combination methods [7], [12]. Based on previous studies, the GLCM has shown the reliability in transforming the fish images into relevant fish features based on texture, and the BPNN proved competent to perform the best performance in the previous studies.

The contributions of this study are validated by comparing the results of image enhancement based on classification methods, so that it is able to (1) show the effect of image enhancement on classification performance; (2) analyse the relationship between image quality and image classification performance; (3) determine the required parameters to achieve the best performance results in the classification model, and (4) present the results of the best classification model to recognize the underwater fish images.

The contents of this paper are organized as follows: In Section 2, previous studies on this research are highlighted. Section 3 presents our proposed model. Section 4 describes the design of our experiment. Subsequently, in Section 5 describes the results of the experiment and discussion. Finally, we draw a conclusion in Section 6. 


\section{Previous studies}

Marine and fisheries biologists have been working for years to improve accuracy of identifying marine fish species. However, almost all of them have considered merely on improving image quality or classification, such as Storbeck and Daan [4], Lee et al. [17], Nery et al. [18], White et al. [19], Rova et al. [20], Larsen et al. [21], Khotimah et al. [22], Iswari et al. [23]. The fish classification performed by Storbeck and Daan [4] used the BPNN method to develop automatic classification of marine fish species based on their shapes. They sorted the fish using a camera that was placed in perpendicular position and a laser source to project the laser-line to the fish that was delivered using conveyor belts. They achieved 95\% accuracy in classifying fish. Lee et al. [17] identified and monitored 22 images of fish species based on their similarities in contours and shapes. Nery et al. [18] used several visual features such as size, shape, color, and texture of fish to identify six fish species based on the Bayesian classification method.

Moreover, White et al. [19] used the same features to locate and to measure several fish species based on discriminant analysis methods. Rova et al. [20], used the linear kernel method based on SVM to identify four different fish species with form features found on the Canny edge detection method and texture features with the $90 \%$ accuracy. Larsen et al. [21], identified three species of cod, haddock, and whiting using the linear regression method to obtain good classification performance with an accuracy of $76 \%$. Khotimah et al. [22] identified tuna species using the Decision Tree (DT) method based on features of colors, shapes, and textures with the accuracy of $88 \%$. Iswari et al. [23] identified fish species using the K-Nearest Neighbor (KNN) method based on color features with an accuracy of $91.36 \%$.

There have been only a few studies that tested the ability to enhance the quality of the image toward determining identification methods, such as Li and Hong's [24], Sengar et al. [25], Hossain et al. [26], and Gu et al. [3]. Li and Hong [24] used image processing to separate the foreground and the background while increasing the contrast in the foreground image. They used a combination of contour extraction and principal component analysis (PCA) for feature engineering and a combination of the Fisher and the Mahalanobis distance model as a classification method. Sengar et al. [25] modified color space and segmentation as an image processing technique for fish identification. The identification was performed manually with three ranges of freshness. However, the dataset was not disrupted by the underwater environment background.

Meanwhile, several studies using fish datasets from marine environments were carried out by Hossain et al. [26] and Gu et al. [3]. Hossain et al. [26] proposed an automatic marine identification for video image dataset using background subtraction method to detect moving objects, pyramid histogram of visual words (PHOW), and Support Vector Machine (SVM) as their classification method. A similar method was performed by Gu et al. [3], with DCP technique to improve the quality of underwater images from disruption of color dominance. The quality of images was improved using the aggregating superpixels technique. They also applied template matching using the inner-distance shape context to search fish in underwater imagery.

One of prior studies that improved image quality was conducted by Iqbal et al. [9], who integrated color models based on contrast values and applying unsupervised color-correction technique and color balancing in contrast correction. Meanwhile, Pujiono et al. proposed the contrast limited adaptive histogram equalization (CLAHE) technique to provide contrast constraints adaptively [8]. Pramunendar et al. [5] proposed the auto level color correction (ALCC) to provide the adaptive contrast in several shades of color so that noise did not appear in all pictures. Several image enhancement techniques as mentioned above yielded low performance if the object was occluded with the dominant color. However, this problem was solved by Carlevaris-Bianco et al. by using the DCP technique to remove haze in the picture caused by dominant colors [10]. The DCP technique was used to estimate ambient light so that color correction using contrast could improve the quality of underwater images [27]. A research performed by Weh et al. [11] determined the dark channels using green and blue channels in underwater photos. Galdran et al. [13] used the DCP technique to reverse the faded red color in underwater images. Furthermore, Borker and Bonde [14] used PCA fusion to enhance contrast and optimize visibility as well as retaining natural appearances. Meanwhile, Kaur and Mahajan [12] combined the DCP technique and the CLAHE technique to improve the distorted image contrast, but it still generated excessive noise. Therefore, Pramunendar et al. [7] used an NCACC to obtain better quality with less noise.

However, these prior studies did not describe the association between the performance of improved image quality and the classification performance 
using the improved data. Most of the image processing techniques were done to separate objects and background images; the background images were arranged in such a way to obtain fish objects quickly. On the other hand, some other researchers did not apply image processing in their research. Some studies only focussed on the feature extraction method, the classification method, or the dataset used.

\section{The proposed approach}

Some studies showed that the performance of the classification methods is influenced by the data, the features, and the classification method. The performance of the classification method shows that color, shape, and texture features do not always cause poor accuracy. These features can be used to display image details precisely based on the equipped functions. However, these features are influenced by data sources. To overcome this research gap, this study proposes a new workflow of fish species identification with improvement on the quality of data sources (see Fig. 2). This study implements the novel contrast-adaptive color correction (NCACC) technique proposed by the authors in the previous work [7] to enhance image

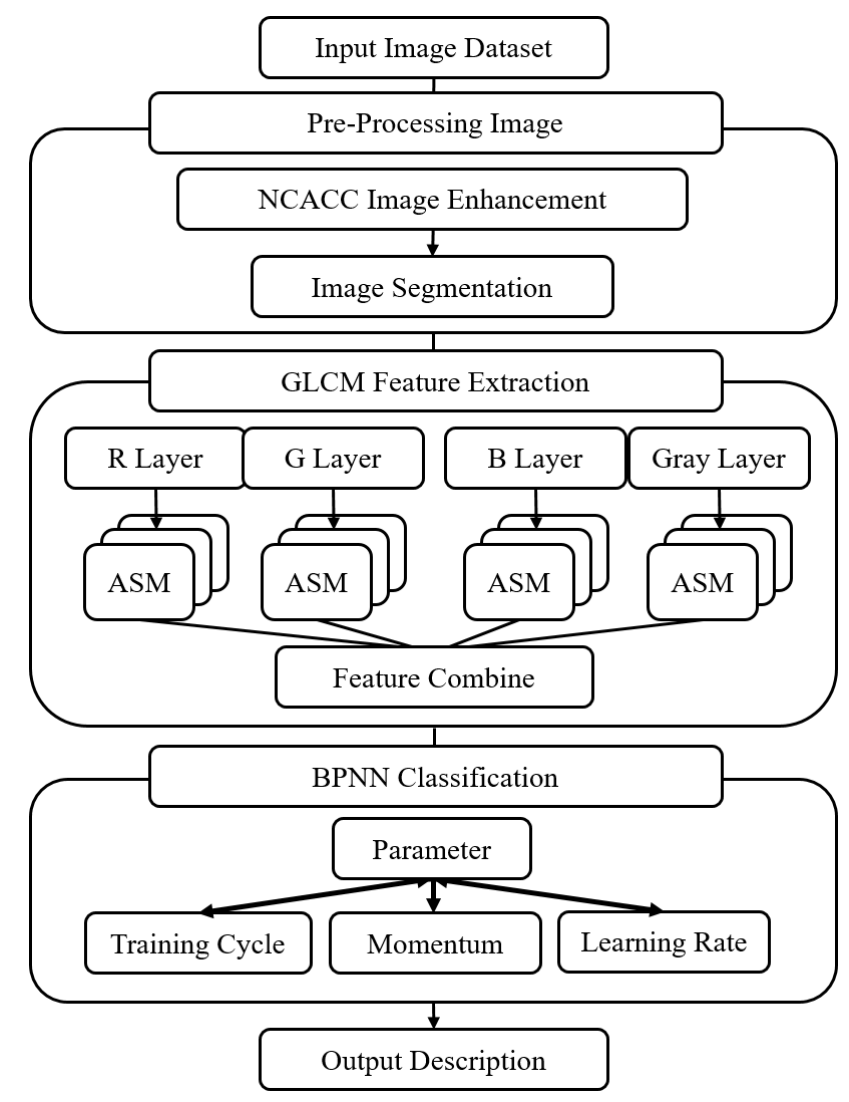

data, which are then transformed the image into useful features such as angular second moment, contrast, correlation, homogeneity, and entropy. These features are obtained by using the gray-level co-occurrence matrix (GLCM) method [28], and the image data are then classified using the backpropagation neural network classification method (BPNN).

\subsection{Image dataset}

In this study, we assume that marine conditions in Indonesia are similar to those of other locations in the world where the objects are in an environment with low light. In addition, the underwater environment also presents other problems that are resolved with the proposed method. The dataset of underwater images is obtained from LifeCLEF 2014 (LCF-14) or Fish4Knowlege image dataset (http://groups.inf.ed.ac.uk/f4k/) [29].

\subsection{Pre-processing image using NCACC}

The pre-processing stage used in this approach model is the novel contrast-adaptive colorcorrection (NCACC) technique. The NCACC technique is a combination of dark channel prior (DCP), auto level color correction (ALCC), and contrast limited adaptive histogram equalization (CLAHE), which is used to improve underwater image quality that is susceptible to bright color distortion and various noise that afflict underwater objects. Based on Pramunendar et al. [7], the stages in the NCACC technique are as follow:

- Estimating the transmission map by applying the lowest and the highest light intensity in the local area from the effects of fog on underwater images.

- Applying filtering techniques and soft image matting techniques to improve transmission maps on white balancing problems.

- Updating the radians value after the recovery process has taken place.

- Estimating ambient light based on the estimated transmission.

- Applying the CLAHE and ALCC techniques at the same time.

The obtained best quality images are manually segmented based on the model of the owned ground truth to generate a fish image without a background. This process was completed for each step of image processing, and everything is applied and tested in the classification process.

Figure. 2 The proposed workflow of fish species identification 


\subsection{Gray-level co-occurrence matrix feature}

Feature extraction transforms the raw image data into numerical data that contain the image's characteristics. The characteristics of the image are critical components of human visual perception, which are obtained based on color, shape, and texture. One method that can be used to do this is to use gray-level co-occurrence matrix (GLCM)[22], [28].

The GLCM method transforms the image into a number based on its textural characteristics, which uses a matrix consisting of $k$ number of rows and $l$ columns at gray-level. GLCM does not depend on variations in image data size. Therefore, this method is suitable for a feature extraction technique. Moreover, GLCM is able show varieties of each fish species' features in the form of numbers according to what is seen by experts.

GLCM represents the relationship between neighboring pixels in the image. The direction of orientation and spatial distance that has been determined gives the new matrix value in the form of the GLCM matrix. The orientation directions in computational GLCM are $0^{\circ}, 45^{\circ}, 90^{\circ}$, and $135^{\circ}$. The results of the GLCM matrix are the co-occurrence matrix and it can be used to obtain angular secondmoment features, contrast, correlation, homogeneity, and entropy $[22,28]$.

The five features are obtained from the image at the 8-bit gray-level from one orientation angle while each layer in the grayscale image is used to get different features. Therefore, when a combination of all image layers and orientation angles are used, it generats 80-feature elements from different segments and angles. The elements consist of 20 characteristics of layer $\mathrm{R}, \mathrm{G}$, and $\mathrm{B}$, while the remaining 20 are obtained from grayscale images. Each image produces 80 features. Therefore, this technique generates a new matrix of 27,370 images multiplied by 80 features.

\subsection{Back-propagation propagation network classification process}

The back-propagation propagation network (BPNN) method us used to test the results of image enhancement by classifying each image consisting of various types of fish based on the Fish4Knowledge dataset. BPNN is a supervised learning algorithm, which includes the training phase and the testing phase. BPNN requires several parameters such as layer number, weight value, bias value, learning rate, value momentum, training cycle, and fault function. All parameters are used in the training phase of the BPNN method. The parameters affect the accuracy in the classification process.

BPNN method is divided into three layers, namely the input layer, hidden layer, and output layer. In the input layer, the data are in a multivariate type. The data used in the input layer are obtained from the feature process in the form of a matrix with the size of $N$ data multiplied by $m$ features. This study uses a matrix with a volume of 27,370 data multiplied by 80 features. The matrix is processed in a hidden layer as shown in Eq. (1), where the hidden layer activates the sigmoid function. The total neurons used are obtained from the average of the total number of attributes and the number of classes that are then added to 1 . The calculation results of the hidden layer process are the result in the output layer (see Eq. (2)). The results of the output layer are then compared with the original label. The match of the analyzed results shows the level of accuracy or the objective value of the BPNN method (see Eq. (3)). In the process, prediction errors are used to update each current weight, as shown in Eq. (4) and Eq. (5). This method updates each of its weights based on Eq. (6) and Eq. (7). BPNN method stops when the repetition is equal to the number of training cycles or the error rate or the expected objective.

$$
\begin{aligned}
& z=b_{i}+\sum_{i=0}^{n} x_{i} v_{i j} \\
& y=b_{0}+\sum_{i=0}^{n} x_{i} w_{i j} \\
& \delta_{y}=\left(t_{k}-z_{k}\right) f^{\prime}(y) \\
& \Delta v=\sum_{j=1}^{m} \delta_{y} w_{i j} \\
& \Delta w=a \delta_{y} z \\
& w_{\text {new }}=w_{\text {old }}+\Delta w \\
& v_{\text {new }}=v_{\text {old }}+\Delta v
\end{aligned}
$$

With $x$ as the input for $z$ in the form of multivariate data. $z$ is a hidden layer obtained from the input process and used as input to $y$ value. Meanwhile, $b$ is the value of bias, $v$ and $w$ are the respective weights of input and hidden layers, respectively. $\Delta v$ and $\Delta w$ are modifications of weights of input and hidden layers, respectively. In addition, $v_{\text {new }}, w_{\text {new }}, v_{\text {old }}$, and $w_{\text {old }}$ are the respective new and old weights of input and hidden layers. The $\alpha$ and $\delta$ are the particular levels of learning and the results of prediction errors obtained from the difference between $t$ and $z$. 


\subsection{Performance evaluation}

Performance evaluation of the classification method is conducted by calculating the accuracy and Kappa. Accuracy is defined as the correct classification of all obtained data. The accuracy value is obtained using Eq. (8) with $t$ and $n$ as the respective numbers of sample data correctly classified, and $n$ as the total number of sample data.

$$
\text { accuracy }=t / n \times 100
$$

Cohen's Kappa coefficient is a measurement of agreement between several variables that can be used to compare the ability of different levels to classify subjects into one of several groups. This value is obtained by using Eq. 9, with $p_{o}$ as an agreement between experts (similar to accuracy) obtained by using Eq. 8 , with $p_{e}$ as the probability of an accidental agreement that is obtained using Eq. 10 . For category $k, N$ is the total data and $n_{k i}$ is the number of categories predicted by experts $k$.

$$
\begin{gathered}
\text { kappa }=\frac{p_{o}-p_{e}}{1-p_{e}} \\
p_{e}=\frac{1}{N^{2}} \sum_{k} n_{k i}
\end{gathered}
$$

\section{Experiment design}

This study shows that data sources are essential to get the best accuracy performance. The previously discussed techniques were applied to the fish4knowledge dataset, and they were tested in a classification process, so that the accuracy of the classification result was based on the improved data. To observe the best accuracy, this study implemented several pre-processing techniques such as dark channel prior (DCP), auto level color correction (ALCC), contrast limited adaptive histogram equalization (CLAHE), auto white balance (AWB), gamma correction (GC), and a combination of several technique as suggested in Kaur and Mahajan [12], such as such as CLAHEDCP, AWBDCP, GCDCP, and ALCCDCP. All of the methods were tested in the previous study [7], which were then compared with the proposed image enhancement technique (NCACC) by comparing the accuracy of each technique. The setting parameter for images enhancement used default values from previous studies [7].

After the quality of image data was improved, it was then transformed into features using the graylevel co-occurrence matrix (GLCM) method. The entire features generated from the GLCM method were 80 features for each image. One image was processed into four color layers namely red, green, blue, and grayscale. Each color layer was divided into four angles based on the GLCM parameters, and each angle was managed into five different features, i.e., angular second moment, contrast, correlation, homogeneity, and entropy. The generated features were processed using the backpropagation neural network (BPNN) method. The best performance results were obtained based on three parameters of the BPNN method, namely momentum, learning rate, and training cycle. The results of the best parameter values were compared with the other image enhancement methods with the other classification method based on previous study.

To evaluate the performance of the classification method, the sampling method and the validation in this process used the default value, which were stratified sampling for sampling methods and 10fold cross-validation. The dataset was divided into ten values for the cross-validation, so that each part was evenly distributed [30]. The experiment was repeated 10 times according to the number of fold cross-validation, and the average results were obtained from the training process and classification performance testing. Performance evaluation was accomplished after a confusion matrix was generated for the classification model, which was used to achieve the accuracy. This study used MATLAB student version 9.6.0.10 (www.mathworks.com) to perform the image enhancement and feature extraction. In addition, RapidMiner version 9.2 (rapidminer.com) was used to perform fish species classification.

\section{Results and discussion}

The proposed model are divided into three steps consisting of pre-processing, feature extraction, and classification (as shown in Fig. 2). The output of each process can be displayed at every stage of the research.

\subsection{Pre-processing image}

The image was enhanced and segmented as described in Table 1. Pramunendar et al. [7] explained that the NCACC technique could improve image quality by $4.4 \%$ compared to some techniques suggested in Kaur and Mahajan [12]. The possible reason is that the NCACC technique is able to show the characteristics of an object by reducing the noise adaptively so that it can show differences between objects. The NCACC method is superior because without adding the filtering method [12]. 
Table 1. Pre-processing image based on [7] and [12]

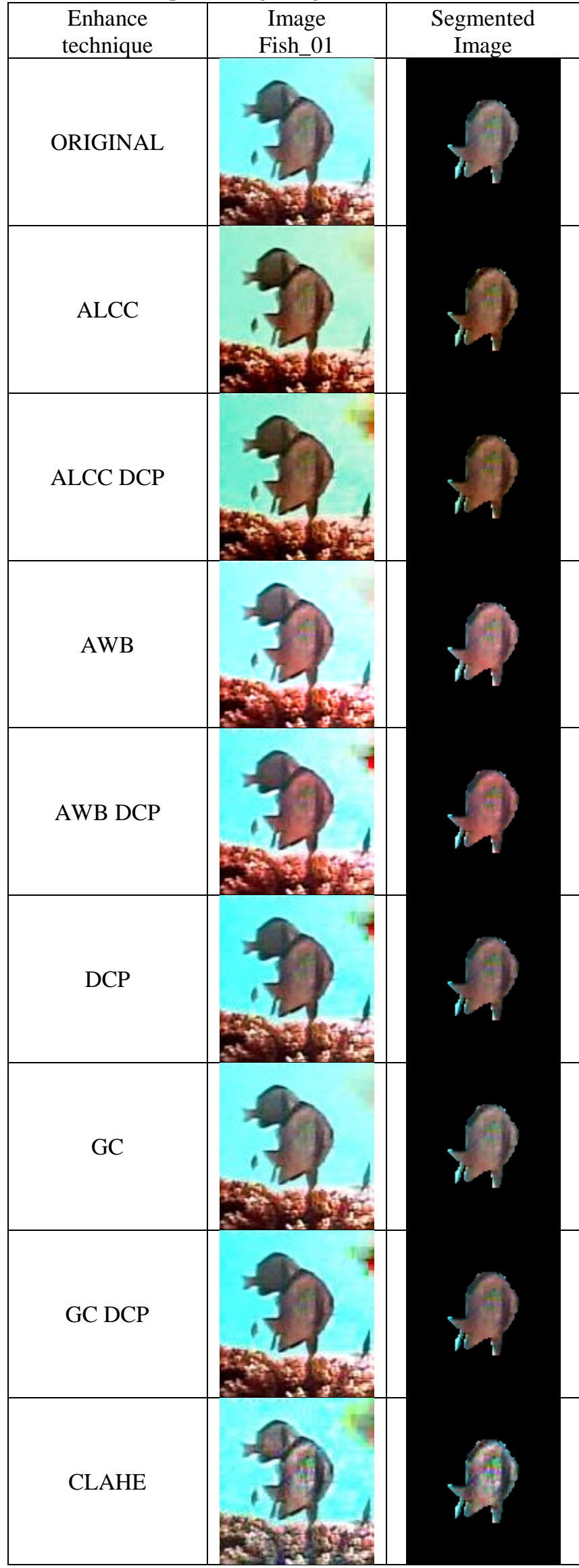

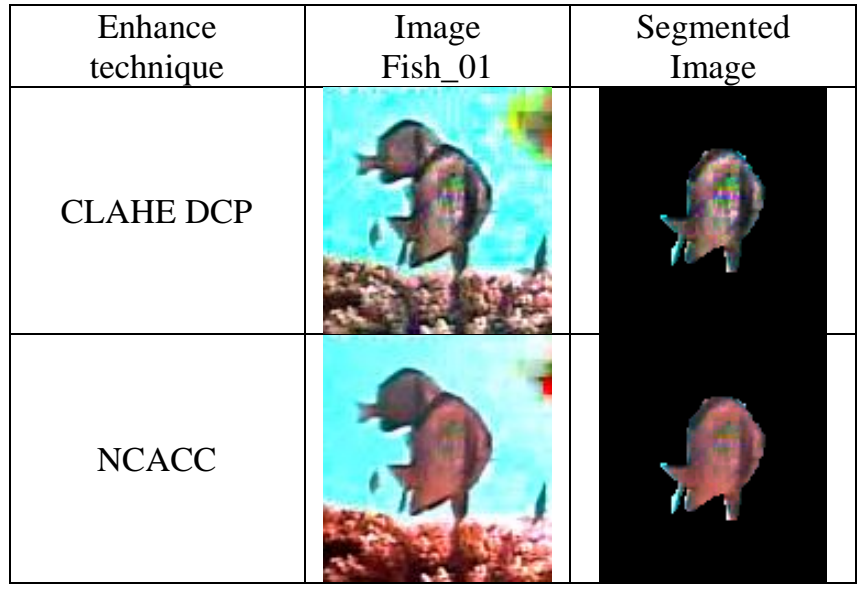

\subsection{Feature extraction}

After the image had been enhanced, the next step in the proposed workflow (Fig. 2) was transforming the image into features. This stage was done using the GLCM method to generate a new matrix of 27,370 images x 80 features. Each row represented data, and the matrix column expressed the features of each information.

As shown in Table 2, there is a column containing variables $X 1, X 2$, until $X 80$, which are the variable containing the respective value of the angular second moment, contrast, and correlation with the orientation direction of 0 degree on the red layer. Meanwhile, the other $X n$ features are a combination of the orientation direction and color layer. Subsequently, the feature $X 80$ is an entropy value with the orientation direction of 135 degrees in the grayscale layer. The matrix in lines 1 to 27,370 is the data number obtained from the dataset. In this study, we used all the features to carry out the classification process so that we assumed all features contributed to getting the best classification performance.

Table 2. Feature extraction applied to original data without resolution enhancement

\begin{tabular}{cccccccc}
\hline No & X1 & X2 & X3 &. &. &. & X80 \\
\hline 1 & 0.628 & 310.6 & 0.824 &. &. &. & 1.904 \\
2 & 0.626 & 199.5 & 0.825 &. &. &. & 1.940 \\
3 & 0.639 & 236.8 & 0.832 &. &. &. & 1.890 \\
4 & 0.642 & 123.1 & 0.850 &. &. &. & 1.914 \\
5 & 0.631 & 83.01 & 0.851 &. &. &. & 1.917 \\
6 & 0.726 & 317.2 & 0.869 &. &. &. & 1.607 \\
7 & 0.719 & 376.2 & 0.867 &. &. &. & 1.673 \\
. &. &. &. &. &. &. &. \\
27,370 & 0.701 & 23.7 & 0.885 &. &. &. & 1.483 \\
\hline
\end{tabular}




\subsection{Evaluation using several classification methods}

In several studies, features were obtained from the images including a selection of a classifier method that was not guided. Normally they used a set of classifiers to be applied to the features and to keep the maximized value of accuracy on the data. This study used some classification methods to evaluate the extracted features of the image enhancements and to compare their results on the original image data that were not enhanced. The classification methods were Support Vector Machine (SVM) [15, 20], Linear Discriminant Analysis (LDA) [21], Naïve Bayes (NB) [18], $k$ Nearest Neighbour (KNN) [23], and Decision Tree (DT) [22].

The parameters for the experiment were determined based on the default value of the tool we used, with details as follows: (1) The best settings for the SVM method were ranging from 0.1 to $0.9,1$ to 9 and 10 to 100 for the SVM C-SVC type operator with the RBF kernel and the penalty parameter of the error term using the value 10; the best parameter using SVM C-SVC for the data after image enhancement value was 10, but for the data without enhancement was 50; (2) Parameters for the KNN method were $k$ value ranged from 1 to 23 and the several distance methods. The best accuracy for $\mathrm{k}$ and distance parameter for the data using image enhancement was 9 and the distance method was maxproductsimilarity. However, the best accuracy for $\mathrm{k}$ and the distance parameter for the data without image enhancement was 7 with the method distance using dicecimilarity; (3) The setting parameter of the DT method were gained ratio for splitting, with minimal size of split and minimal leaf note was 4 and 2, respectively. The DT was confident with the amount of $9.5 \%$, and the minimal gain was 0.1 ; and (4) the setting parameter for BPNN method were a range from 0.1 to 1 for momentum and learning rates, but the training cycle was configured to be 100 to 1000 cycles. The best value of parameter for momentum, learning and training cycle is $0.1,0.1$ and 1000, respectively. The value of the hidden layer was 53 .

Table 3 and 4 show that image enhancement using the NCACC technique improved the accuracy of each classification method. The highest performance accuracy for classification using the NCACC technique was obtained using the BPNN method (93.73\%). Performance evaluation using Kappa also showed that BPNN was the best classification method of 0.92 .
Table 3. Accuracy value of several classification methods based on the NCACC enhancement technique

\begin{tabular}{|c|c|c|c|}
\hline Method & $\begin{array}{c}\text { Accuracy of } \\
\text { Original Data }\end{array}$ & $\begin{array}{c}\text { Accuracy of } \\
\text { Enhanced Data }\end{array}$ & Increase \\
\hline BPNN & $89.05 \%$ & $93.73 \%$ & $4.68 \%$ \\
\hline DT & $44.91 \%$ & $64.03 \%$ & $19.12 \%$ \\
\hline KNN & $66.79 \%$ & $72.96 \%$ & $6.17 \%$ \\
\hline LDA & $78.11 \%$ & $89.67 \%$ & $11.56 \%$ \\
\hline NB & $15.99 \%$ & $27.96 \%$ & $11.98 \%$ \\
\hline SVM & $77.84 \%$ & $79.66 \%$ & $1.82 \%$ \\
\hline
\end{tabular}

Table 4. Kappa value of several classification methods based on the NCACC enhancement technique

\begin{tabular}{|c|c|c|c|}
\hline Method & $\begin{array}{c}\text { Kappa of } \\
\text { Original Data }\end{array}$ & $\begin{array}{c}\text { Kappa of } \\
\text { Enhanced Data }\end{array}$ & Increase \\
\hline BPNN & 0.87 & 0.92 & 0.05 \\
\hline DT & 0.02 & 0.44 & 0.42 \\
\hline KNN & 0.53 & 0.63 & 0.10 \\
\hline LDA & 0.69 & 0.75 & 0.06 \\
\hline NB & 0.11 & 0.20 & 0.09 \\
\hline SVM & 0.59 & 0.69 & 0.10 \\
\hline
\end{tabular}

By using the appropriate parameters obtained from the experiment, both Table 3 and 4 provide the same pattern of the evaluation results. The highest classification increment was achieved by the DT method with increment of accuracy up to $19.12 \%$. The Kappa value also showed the highest performance increment up to 0.4. This DT method and the parameters are suitable if they are applied to the fish4knowledge dataset, given that they are implemented with the NCACC enhancement method. The other classification method that also gained improvement of accuracy and Kappa value was the NB method. NB obtained accuracy improvement up to $11.98 \%$ and Kappa value up to 0.1 . However, the NB classification method was less suitable for classifying the fish4knowledge dataset even though the image quality had been enhanced as the accuracy was still lower than 50\%. In our estimation, the performance of the NB method declined when the numbers of features were increased and the features used the real value.

Different from the NB method, the LDA classification method obtained an accuracy of $78.11 \%$ using original data and an increment of $11.56 \%$ to be $89.67 \%$ using the enhanced data, even though the Kappa value of the LDA method only increased 0.6 points from 0.69 to 0.75 . The LDA method accuracy increased similar to the NB method, but its accuracy was better than the NB method because the NB method selecting a class that has the greatest probability. While the probability of the NB method is not correlated with the correct classification label that causes by 
independent features each other and sometimes has the same data. In the other side, LDA to project the data by linear combination forms of all the features. The combination is not allowed each the features correlate with each other, yet increases the correlation with the correct classification label. This causes the LDA method to be superior to the NB method. On the other hand, the increase of accuracy and Kappa value were also obtained through KNN and SVM methods. This increase in accuracy value was up to $6.17 \%$ through $\mathrm{KNN}$ and $1.82 \%$ through SVM. The Kappa value also increased up to 0.1 for KNN and SVM method. That increase was obtained because we applied NCACC for image enhancement methods so the image looks clearer without looking blurry like fog. While the classification method works according to its capabilities, and the BPNN method is superior to all methods in the experiment. The BPNN method mapping the inputs where the inputs can be separated by a classifier without finding the separator between classes, and the features in the BPNN method is correlated with the correct classification label.

\subsection{Parameters evaluations of BPNN classification using the original data}

The performance of the BPPN method applied to the original dataset is shown in Table 5 to 7 . Table 5 shows that an increase in the number of training cycles affects the performance of accuracy. There are several values of performance in indicating an improvement, but they are still below the average resulted from each experiment. The increasing of performance is caused an increase in training cycles so that the iterative adjustment of the weights that happens. The weights are optimized among neurons through the backward propagate of the error.

Meanwhile, the best performance was obtained using training cycles with the values of 1000 , which is the highest value within the limits of the experiment. These parameters achieved the accuracy of $89.05 \%$ and the Kappa value of 0.858 . The settings parameter provided a $15 \%$ increase compared with the use of 10 training cycle parameter values with an average rise of the accuracy by $0.833 \%$ per trial cycle. Therefore, it can be concluded that the more training cycle conducted, the higher the accuracy performance.

Increasing the value of the learning rate cause the model to converge quickly to a suboptimal solution and reduces the performance. This is shown in Table 6, where the highest accuracy and the Kappa value were obtained when the best learning
Table 5. The accuracy and Kappa value of BPPN classification based on training cycle parameter

\begin{tabular}{|c|c|c|c|c|}
\hline $\begin{array}{c}\text { Training } \\
\text { Cycle }\end{array}$ & $\begin{array}{c}\text { Learning } \\
\text { Rate }\end{array}$ & $\begin{array}{c}\text { Momen } \\
\text { tum }\end{array}$ & $\begin{array}{c}\text { Accuracy } \\
(\%)\end{array}$ & Kappa \\
\hline 10 & 0.1 & 0.1 & 74.05 & 0.632 \\
\hline 20 & 0.1 & 0.1 & 78.45 & 0.701 \\
\hline 30 & 0.1 & 0.1 & 79.71 & 0.714 \\
\hline 40 & 0.1 & 0.1 & 81.02 & 0.737 \\
\hline 50 & 0.1 & 0.1 & 82.36 & 0.758 \\
\hline 60 & 0.1 & 0.1 & 83.20 & 0.769 \\
\hline 70 & 0.1 & 0.1 & 83.02 & 0.767 \\
\hline 80 & 0.1 & 0.1 & 84.41 & 0.786 \\
\hline 90 & 0.1 & 0.1 & 84.16 & 0.781 \\
\hline 100 & 0.1 & 0.1 & 84.65 & 0.791 \\
\hline 200 & 0.1 & 0.1 & 86.30 & 0.814 \\
\hline 300 & 0.1 & 0.1 & 86.96 & 0.821 \\
\hline 400 & 0.1 & 0.1 & 87.72 & 0.834 \\
\hline 500 & 0.1 & 0.1 & 87.88 & 0.836 \\
\hline 600 & 0.1 & 0.1 & 88.52 & 0.844 \\
\hline 700 & 0.1 & 0.1 & 88.23 & 0.840 \\
\hline 800 & 0.1 & 0.1 & 88.98 & 0.851 \\
\hline 900 & 0.1 & 0.1 & 88.74 & 0.847 \\
\hline 1000 & 0.1 & 0.1 & 89.05 & 0.858 \\
\hline & & & & \\
\hline
\end{tabular}

Table 6. The accuracy and Kappa value of BPPN classification based on learning rate parameter

\begin{tabular}{|c|c|c|c|c|}
\hline $\begin{array}{c}\text { Training } \\
\text { Cycle }\end{array}$ & $\begin{array}{c}\text { Learning } \\
\text { Rate }\end{array}$ & $\begin{array}{c}\text { Momen } \\
\text { tum }\end{array}$ & $\begin{array}{c}\text { Accuracy } \\
(\%)\end{array}$ & Kappa \\
\hline 1000 & 0.1 & 0.1 & 89.05 & 0.858 \\
\hline 1000 & 0.2 & 0.1 & 88.47 & 0.844 \\
\hline 1000 & 0.3 & 0.1 & 87.88 & 0.835 \\
\hline 1000 & 0.4 & 0.1 & 87.69 & 0.833 \\
\hline 1000 & 0.5 & 0.1 & 82.55 & 0.761 \\
\hline 1000 & 0.6 & 0.1 & 67.17 & 0.555 \\
\hline 1000 & 0.7 & 0.1 & 59.83 & 0.463 \\
\hline 1000 & 0.8 & 0.1 & 62.18 & 0.486 \\
\hline 1000 & 0.9 & 0.1 & 48.71 & 0.338 \\
\hline 1000 & 1 & 0.1 & 49.19 & 0.323 \\
\hline
\end{tabular}

rate was 0.1. Furthermore, as the learning rate increases, the accuracy declines until it reaches the level of $49.19 \%$. These results have been confirmed with Eqs. (4) and (5), in which the increase of the learning rate affects the new weights for the subsequent learning process and influences the classification accuracy.

Table 7 shows that the lowest momentum of 0.1 generates the highest accuracy and Kappa value. As the momentum parameter increases, the accuracy reduces until it reaches the value of $44.26 \%$. These results show that smaller the momentum value brings features closest to various features that have similarities, and without speeding up convergence. 
Table 7. The accuracy and Kappa value of BPPN

classification based on momentum parameter

\begin{tabular}{|c|c|c|c|c|}
\hline $\begin{array}{c}\text { Training } \\
\text { Cycle }\end{array}$ & $\begin{array}{c}\text { Learning } \\
\text { Rate }\end{array}$ & $\begin{array}{c}\text { Momen } \\
\text { tum }\end{array}$ & $\begin{array}{c}\text { Accuracy } \\
(\%)\end{array}$ & Kappa \\
\hline 1000 & 0.1 & 0.1 & 89.05 & 0.858 \\
\hline 1000 & 0.1 & 0.2 & 88.96 & 0.851 \\
\hline 1000 & 0.1 & 0.3 & 89.01 & 0.850 \\
\hline 1000 & 0.1 & 0.4 & 88.83 & 0.849 \\
\hline 1000 & 0.1 & 0.5 & 88.36 & 0.843 \\
\hline 1000 & 0.1 & 0.6 & 88.63 & 0.846 \\
\hline 1000 & 0.1 & 0.7 & 88.40 & 0.842 \\
\hline 1000 & 0.1 & 0.8 & 86.81 & 0.821 \\
\hline 1000 & 0.1 & 0.9 & 50.38 & 0.340 \\
\hline 1000 & 0.1 & 1 & 44.26 & 0 \\
\hline
\end{tabular}

Therefore, the classification performance in obtaining accuracy and Kappa value decreases.

Tables 5 to 7 show that the BPPN is able to achieve accuracy of $89.05 \%$ and the Kappa value of 0.858 . These values are supported by several parameter settings such as the training cycle of 1000 , the learning rate of 0.1 , and the momentum of 0.1 . The results show that there is a classification failure of $10.95 \%$. This failure occurred in data that had a very small number of members and data whose features were similar to other class members. These features were similar because of the dominance of color in the underwater environment, and this made the texture of the object look similar to other classes. The best results were used for the next experiment.

\subsection{Evaluation using BPNN classification method}

The images were enhanced and inputted to the BPNN classification method. In this experiment, we

Accuracy Comparison between Enhance Data for Classification using BPNN

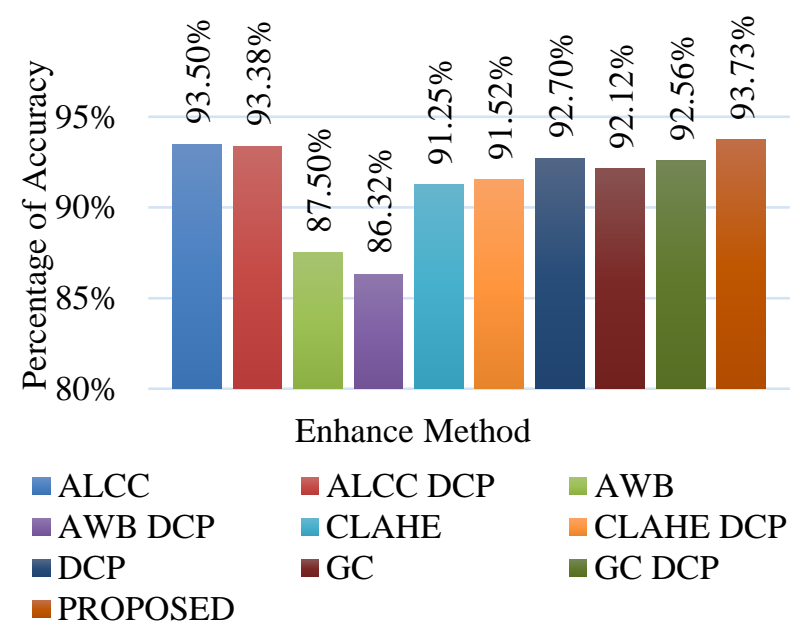

Figure 3. Accuracy Comparison between Enhancement Data for BPNN Classification

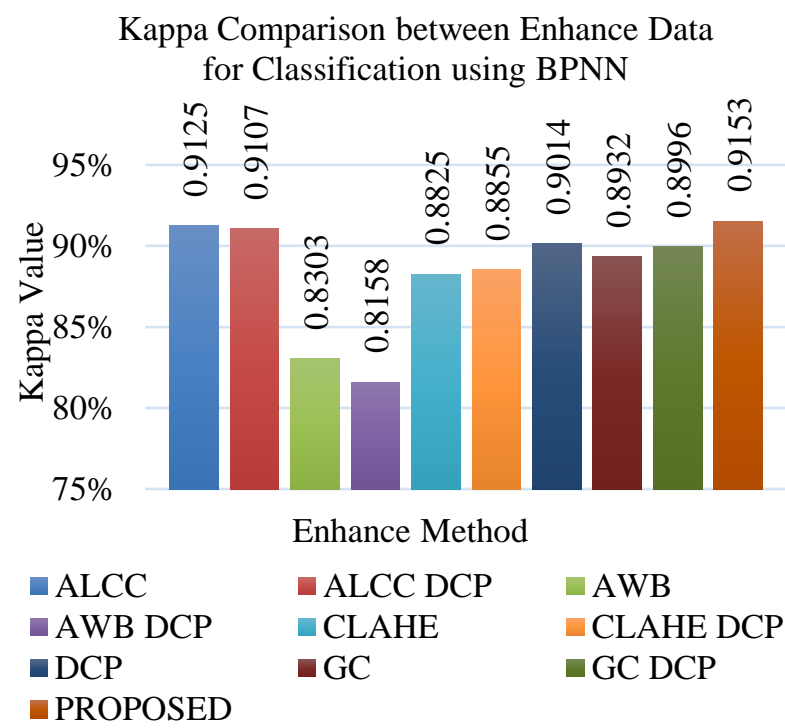

Figure 4. Kappa comparison between enhancement data for BPNN Classification

used the parameter based on the best results from the previous experiment such as training cycle of 1000 cycles, the learning rate of 0.1 , the momentum of 0.1 , stratified sampling method, and 10-fold cross-validation.

In Fig. 3 and 4, the highest accuracy and Kappa value of the tests using the BPNN classification method are obtained using NCACC technique, which are $93.73 \%$ and 0.9153 respectively. The performance of the NCACC technique is higher than the ALCC technique for enhancing the original data. Similar to the previous study [7], the ALCC technique yielded a higher performance than the combination of ALCCDCP, while the AWB was higher than AWBDCP. However, different results occurred in the application of the CLAHE technique with a combination of CLAHEDCP. The results showed that the CLAHE accuracy was lower than CLAHEDCP. In addition, the performance of the GCDCP combination was higher than the GC.

In order to explain the detail of the performance based on each label, we used accuracy without Kappa because the pattern of both performance result was quite similar. The detailed explanation of accuracy of each label is shown in Table 8, in which various image enhancement techniques such as AWB (as seen in E1), CLAHE (as seen in E2), GC (as seen in E3), ALCC (as seen in E4), DCP (as seen in E5), and NCACC (as seen in E6) were applied to the BPNN classification method. Meanwhile, the performance of the BPNN classification using the dataset of the enhanced images using the combined techniques, such as AWBDCP (as seen in E7), CLAHEDCP (as seen in E8), GCDCP (as seen in 
Table 8. BPNN Classification Accuracy of each label based on the enhanced images (in percentage) (where E1 is AWB, E2 is CLAHE, E3 is GC, E4 is ALCC, E5 is

\begin{tabular}{|c|c|c|c|c|c|c|}
\hline \multicolumn{7}{|c|}{$\mathrm{DCP}$, and E6 is NCACC) } \\
\hline label & E1 & E2 & E3 & E4 & E5 & E6 \\
\hline 01 & 93.5 & 93.8 & 96.1 & 95.9 & 96.2 & 96.8 \\
\hline 02 & 88.5 & 90.9 & 89.6 & 92.6 & 91.4 & 92.4 \\
\hline 03 & 87.1 & 89.9 & 87.0 & 93.1 & 91.0 & 91.6 \\
\hline 04 & 80.4 & 96.7 & 96.7 & 98.1 & 96.9 & 97.2 \\
\hline 05 & 93.5 & 96.6 & 97.4 & 97.2 & 97.3 & 98.4 \\
\hline 06 & 26.8 & 36.8 & 39.0 & 44.7 & 33.7 & 55.8 \\
\hline 07 & 84.9 & 86.2 & 88.4 & 89.6 & 87.3 & 89.8 \\
\hline 08 & 41.7 & 41.3 & 46.3 & 46.8 & 47.7 & 44.0 \\
\hline 09 & 70.5 & 75.9 & 73.9 & 77.2 & 73.0 & 76.8 \\
\hline 10 & 98.0 & 98.3 & 98.7 & 98.0 & 97.7 & 99.0 \\
\hline 11 & 24.5 & 19.4 & 33.7 & 35.7 & 21.4 & 35.7 \\
\hline 12 & 44.9 & 57.1 & 59.2 & 65.3 & 54.4 & 56.5 \\
\hline 13 & 66.3 & 76.8 & 77.9 & 82.9 & 79.0 & 87.9 \\
\hline 14 & 22.2 & 23.3 & 34.4 & 31.1 & 23.3 & 34.4 \\
\hline 15 & 40.5 & 33.3 & 35.7 & 66.7 & 52.4 & 47.6 \\
\hline 16 & 79.6 & 77.7 & 83.0 & 80.1 & 78.6 & 81.1 \\
\hline 17 & 38.8 & 65.3 & 73.5 & 87.8 & 63.3 & 67.4 \\
\hline 18 & 78.6 & 76.8 & 83.9 & 87.5 & 75.0 & 83.9 \\
\hline 19 & 51.7 & 55.2 & 62.1 & 34.5 & 51.7 & 69.0 \\
\hline 20 & 4.8 & 9.5 & 14.3 & 33.3 & 33.3 & 28.6 \\
\hline 21 & 0 & 0 & 0 & 6.3 & 0 & 6.3 \\
\hline 22 & 7.3 & 19.5 & 22.0 & 31.7 & 22.0 & 46.3 \\
\hline 23 & 56.0 & 76.0 & 88.0 & 88.0 & 84.0 & 80.0 \\
\hline
\end{tabular}

E9), and ALCCDCP (as seen in E10) can be seen in Table 9.

The NCACC technique (E6) in Table 8 and 9 yielded an accuracy of more than $85 \%$ found on the labels fish 01, 02, 03, 04, 05, 07, 10, and 13 with the number of images on those labels is 25,901 images. While the rest is only 1,469 images that produce accuracy below $85 \%$. From 25,901 images only on label fish 13 that has the smallest number of images that are 181 images and provide accuracy $87.85 \%$ equivalent with 22 incorrect images label, while the others have fewer incorrect images label.

In the remaining labels, the accuracy was lower than $85 \%$, showing that not all species were well identified. The total data for this label were 1469 images consisting of 15 different species, and only 869 or $59 \%$ images could be identified. The highest number of images of the 15 species was in fish_09 species with the total data of 241 images, and only
Table 9. BPNN Classification Accuracy of each label

based on the combination enhanced images (in percentage) (where E6 is NCACC, E7 is AWBDCP, E8 is

CLAHEDCP, E9 is GCDCP, and E10 is ALCCDCP)

\begin{tabular}{|c|c|c|c|c|c|}
\hline label & E7 & E8 & E9 & E10 & E6 \\
\hline 01 & 90.3 & 95.5 & 96.0 & 96.0 & 96.8 \\
\hline 02 & 87.7 & 90.0 & 91.3 & 92.2 & 92.4 \\
\hline 03 & 88.0 & 89.2 & 91.6 & 93.5 & 91.6 \\
\hline 04 & 82.1 & 95.7 & 96.0 & 97.6 & 97.2 \\
\hline 05 & 94.0 & 97.4 & 97.9 & 97.8 & 98.4 \\
\hline 06 & 22.1 & 33.2 & 31.6 & 43.7 & 55.8 \\
\hline 07 & 88.2 & 88.2 & 87.1 & 90.9 & 89.8 \\
\hline 08 & 37.6 & 38.5 & 43.6 & 47.7 & 44.0 \\
\hline 09 & 70.5 & 71.0 & 72.2 & 74.3 & 76.8 \\
\hline 10 & 97.7 & 98.3 & 98.7 & 98.7 & 99.0 \\
\hline 11 & 17.4 & 11.2 & 18.4 & 21.4 & 35.7 \\
\hline 12 & 42.2 & 55.1 & 51.7 & 57.1 & 56.5 \\
\hline 13 & 56.9 & 66.3 & 75.7 & 80.7 & 87.9 \\
\hline 14 & 24.4 & 15.6 & 33.3 & 22.2 & 34.4 \\
\hline 15 & 42.9 & 38.1 & 52.4 & 57.1 & 47.6 \\
\hline 16 & 81.6 & 76.7 & 82.5 & 82.5 & 81.1 \\
\hline 17 & 46.9 & 67.4 & 46.9 & 65.3 & 67.4 \\
\hline 18 & 75.0 & 73.2 & 82.1 & 76.8 & 83.9 \\
\hline 19 & 65.5 & 51.7 & 62.1 & 44.8 & 69.0 \\
\hline 20 & 4.8 & 23.8 & 23.8 & 28.6 & 28.6 \\
\hline 21 & 0 & 0 & 6.3 & 0 & 6.3 \\
\hline 22 & 4.9 & 14.6 & 34.2 & 63.4 & 46.3 \\
\hline 23 & 52.0 & 72.0 & 92.0 & 80.0 & 80.0 \\
\hline & & & & & \\
\hline 04
\end{tabular}

185 or $77 \%$ images could be identified. There were ten labels with total data under 100 images, but fish 21 used the smallest number of images with 16 images, and only 1 or $6 \%$ images could be identified; this value allowed the image to be a datum that was used as training.

In Table 8 and 9, only NCACC (E6), ALCC (E4), and GCDCP (E9) could recognize the image labelled fish 21 (Neoglyphidodon Nigroris), whereas most of the other methods recognized it as fish 01 . Some fish images labelled as fish 21 that were identified using the BPNN classification method showed that the proposed image enhancement technique did not visually change all of the appearances of the image, yet provide sharpened in parts of image appearance (see Table 10).

Based on these results, it can be concluded that the accuracy in the classification process is influenced by image enhancement. Moreover, accompanied by the use of appropriate classification 
Table 10. The Fish $\_21$ image that was recognized

\begin{tabular}{|c|c|c|}
\hline $\begin{array}{c}\text { Enhance } \\
\text { technique }\end{array}$ & Original Image & Enhanced Image \\
\hline GCDCP & & \\
\hline ALCC & & \\
NCACC & & \\
\hline
\end{tabular}

parameters, the classification performance can be improved. Therefore, problems that occur in underwater environment and create obstacles in the classification process can be solved by applying image enhancement. Based on our experiment, we show that the NCACC technique is one of the best techniques for this problem.

\section{Conclusions}

Protecting the populations of endangered aquatic biota is our intention and we might have solved one of the hardest challenges faced by many countries. However, some automated fish identification techniques have not considered the issues in the original data even though some data were taken from various environmental conditions. This study proposes a new workflow of fish species identification that is expected to provide a high level of accuracy when the proposed method is applied to classify underwater fish images. Our model improves the accuracy by $4.68 \%$ and Kappa value by 0.05 implemented on 27,370 images consisting of 23 fish species. These results indicate that our proposed can improve accuracy as it enhances the quality of the original underwater images. An accuracy improvement can be made because the characteristics of the object have been improved so that the difference among objects in the image was more distinguishable.

In addition to be tested with the BPNN classification method, the proposed method was also tested with various classification methods. The results show that the NCACC technique enhancement method increased the accuracy of all the tested classification methods. Therefore, it can be concluded that, in addition to the classification and feature extraction methods, the image enhancement method also affects the accuracy of fish species identification. Therefore, the improvement in the pre-processing step is essential as the sharpness of the improved image makes it easier to show the characteristics of each image and subsequently improves the quality of the classification performance.

\section{Acknowledgments}

This work funded by Indonesian Ministry of Research and Higher Learning (DPRM-DIKTI) including supported by the Department of Electrical Engineering and Information Technology, Faculty of Engineering, Universitas Gadjah Mada and Faculty of Computer Science, Universitas Dian Nuswantoro.

\section{References}

[1] World Bank, "Fish species, threatened. World Development Indicators", 2018. [Online]. Available:

https://databank.worldbank.org/data/reports.asp $\mathrm{x}$ ? source $=2 \&$ series $=$ EN.FSH.THRD.NO\&coun try\#.

[2] M. Suganuma, D. Tsuchiya, S. Shirakawa, and T. Nagao, "Hierarchical Feature Construction for Image Classification using Genetic Programming", In: Proc. of IEEE International Conference on Systems, Man, and Cybernetics, pp. 001423-001428, 2016.

[3] Z. Gu, R. Wang, J. Dai, H. Zheng, and B. Zheng, "Automatic Searching of Fish from Underwater Images via Shape Matching", In: Proc. of OCEANS 2016, pp. 1-4, 2016.

[4] F. Storbeck and B. Daan, "Fish Species Recognition using Computer Vision and a Neural Network", Fisheries Research, Vol. 51, pp. 11-15, 2001.

[5] R. A. Pramunendar, G. F. Shidik, C. Supriyanto, P. N. Andono, and M. Hariadi, "Auto Level Color Correction for Underwater Image Matching Optimization", International Journal of Computer Science and Network Security, Vol. 13, No. 1, pp. 18-23, 2013.

[6] Y. Sari and R. A. Pramunendar, "Classification Quality of Tobacco Leaves as Cigarette Raw Material Based on Artificial Neural Networks", International Journal of Computer Trends and 
Technology, Vol. 50, No. 3, pp. 147-150, 2017.

[7] R. A. Pramunendar, S. Wibirama, and P. I. Santosa, "A Novel Approach for Underwater Image Enhancement based on Improved Dark Channel Prior with Colour Correction", Journal of Engineering Science and Technology, Vol. 13, No. 10, pp. 3220-3237, 2018.

[8] Pujiono, N. A. Pulung, I. K. E. Purnama, and M. Hariadi, "Color Enhancement Of Underwater Coral Reef Images Using Contrast Limited Adaptive Histogram Equalization (CLAHE) With Rayleigh Distribution", In: Proc. of International Conferences on Information, Communication, Technology, and Systems, pp. 45-52, 2013.

[9] K. Iqbal, R. A. Salam, A. Osman, and A. Z. Talib, "Underwater Image Enhancement Using an Integrated Colour Model", IAENG International Journal of computer science, Vol. 34, No. 2, p. 12, 2007.

[10] N. Carlevaris-Bianco, A. Mohan, and R. M. Eustice, "Initial Results in Underwater Single Image Dehazing", In: Proc. of OCEANS 2010, pp. 1-8, 2010.

[11] H. Wen, Y. Tian, T. Huang, and W. Gao, "Single Underwater Image Enhancement with a New Optical Model", In: Proc. of IEEE International Symposium on Circuits and Systems, pp. 753-756, 2013.

[12] E. H. Kaur and R. Mahajan, "Improved Dark Channel Prior using Gabor Filter and Clahe", International Journal of Application or Innovation in Engineering \& Management, Vol. 3, No. 7, pp. 127-134, 2014.

[13] A. Galdran, D. Pardo, A. Picón, and A. Alvarez-Gila, "Automatic Red-Channel Underwater Image Restoration", Journal of Visual Communication and Image Representation, Vol. 26, pp. 132-145, 2015.

[14] S. Borker and S. Bonde, "Contrast Enhancement and Visibility Restoration of Underwater Optical Images Using Fusion", International Journal of Intelligent Engineering and Systems, Vol. 10, No. 4, pp. 217-225, 2017.

[15] X. Sun, J. Shi, J. Dong, and X. Wang, "Fish Recognition from Low-Resolution Underwater Images", In: Proc. of 2016 9th International Congress on Image and Signal Processing, BioMedical Engineering and Informatics, pp. 471-476, 2016.

[16] H. Qin, X. Li, Z. Yang, and M. Shang, "When Underwater Imagery Analysis Meets Deep Learning: a Solution at the Age of Big Visual Data", In: Proc. of OCEANS 2015, pp. 1-5, 2015.
[17] D.-J. Lee, R. B. Schoenberger, D. Shiozawa, X. $\mathrm{Xu}$, and P. Zhan, "Contour Matching for a Fish Recognition and Migration-Monitoring System", In: Proc. SPIE 5606, Two- and ThreeDimensional Vision Systems for Inspection, Control, and Metrology II, Vol. 5606, p. 37, 2004.

[18] M. S. Nery, A. M. Machado, M. F. M. Campos, F. L. C. Pádua, R. Carceroni, and J. P. QueirozNeto, "Determining the Appropriate Feature Set for Fish Classification Tasks", In: Proc. of Brazilian Symposium of Computer Graphic and Image Processing, Vol. 2005, pp. 173-180, 2005.

[19] D. J. White, C. Svellingen, and N. J. C. Strachan, "Automated Measurement of Species and Length of Fish by Computer Vision", Fisheries Research, Vol. 80, No. 2-3, pp. 203210, 2006.

[20] A. Rova, G. Mori, and L. M. Dill, "One fish, two fish, butterfish, trumpeter: Recognizing fish in underwater video", In: Proc. of Conference on Machine Vision Applications, pp. 404-407, 2007.

[21] R. Larsen, H. Olafsdottir, and B. K. Ersbøll, "Shape and Texture Based Classification of Fish Species", Image Analysis, pp. 745-749, 2009.

[22] W. N. Khotimah, A. Z. Arifin, A. Yuniarti, A. Y. Wijaya, D. A. Navastara, and M. A. Kalbuadi, "Tuna Fish Classification using Decision Tree Algorithm and Image Processing Method", In: Proc. of International Conference on Computer, Control, Informatics and its Applications, pp. 126-131, 2015.

[23] N. M. S. Iswari, Wella, and Ranny, "Fish Freshness Classification Method based on Fish Image using K-Nearest Neighbor", In: Proc. of the 4th International Conference on New Media Studies, Vol. 2017, pp. 87-91, 2017.

[24] L. Li and J. Hong, "Identification of Fish Species based on Image Processing and Statistical Analysis Research", In: Proc. of IEEE International Conference on Mechatronics and Automation, pp. 1155-1160, 2014.

[25] N. Sengar, V. Gupta, M. K. Dutta, and C. M. Travieso, "Image Processing based Method for Identification of Fish Freshness using Skin Tissue", In: Proc. of the 4th International Conference on Computational Intelligence \& Communication Technology, pp. 1-4, 2018.

[26]E. Hossain, S. M. S. Alam, A. A. Ali, and M. A. Amin, "Fish Activity Tracking and Species Identification in Underwater Video", In: Proc. 
of the 5th International Conference on Informatics, Electronics and Vision, pp. 62-66, 2016.

[27] H.-Y. Yang, P.-Y. Chen, C.-C. Huang, Y.-Z. Zhuang, and Y.-H. Shiau, "Low Complexity Underwater Image Enhancement Based on Dark Channel Prior", In: Proc. of the 2nd International Conference on Innovations in Bio-inspired Computing and Applications, pp. 17-20, 2011.

[28] P. X. Huang, "Hierarchical Classification System with Reject Option for Live Fish Recognition", Intelligent Systems Reference Library, Vol. 104, Cham: Springer International Publishing, pp. 141-159, 2016.

[29] B. J. Boom, P. X. Huang, J. He, and R. B. Fisher, "Supporting Ground-Truth Annotation of Image Datasets using Clustering", In: Proc. of the 21 st International Conference on Pattern Recognition, pp. 1542-1545, 2012.

[30] G. Wang, J.-N. Hwang, K. Williams, F. Wallace, and C. S. Rose, "Shrinking Encoding with Two-Level Codebook Learning for FineGrained Fish Recognition", In: Proc. of the 2016 ICPR 2nd Workshop on Computer Vision for Analysis of Underwater Imagery, pp. 31-36, 2016. 